\title{
Reinforcement-based cognitive biases as vulnerability factors in alcohol addiction: From humans to animal models
}

\author{
Karolina Noworyta ${ }^{1}$, Agata Cieslik$^{1}$, and Rafal Rygula ${ }^{1}$ \\ ${ }^{1}$ Maj Institute of Pharmacology Polish Academy of Sciences
}

March 15, 2021

\begin{abstract}
Alcohol use disorder (AUD) is one of the most common but still poorly treated psychiatric conditions. Developing new treatments requires a better understanding of the aetiology of symptoms and evaluation of novel therapeutic targets in preclinical studies. Recent developments in our understanding of the reinforcement-based cognitive biases (RBCBs) that contribute to the development of AUD and its treatment offer new opportunities for both clinical and preclinical research. In this review, we first briefly describe psychological and cognitive theories that implicate various aspects of reinforcement sensitivity in the development, maintenance, and recurrence of alcohol addiction. Furthermore, in separate sections, we describe studies investigating RBCBs and their neural, neurochemical, and pharmacological correlates, and we discuss possible interactions between RBCBs and trajectories of AUD. Finally, we describe how recent translational studies using state-of-the-art animal models can facilitate our understanding of the role of reinforcement sensitivity and RBCBs in various aspects of AUD.
\end{abstract}

\section{Introduction}

Alcohol addiction is one of the biggest problems of modern society through its impact on health, social cohesion, crime, and its comorbidity with other neuropsychiatric disorders. Major advances in genetics and molecular neurobiology have led to the identification of many of the primary targets of alcohol and revealed neuroadaptations that develop with its chronic consumption. However, understanding pre-existing cognitive deficits that serve as substrates compounding the initiation of alcohol use and the development of alcohol use disorder (AUD) remains a major challenge.

All goal-directed behaviours, including alcohol use and abuse, are composed of two core components: the decision-making process, which starts with representations of the available options that lead to the selection of the option with the highest expected value, and reinforcement learning, through which outcomes are used to refine value expectations. The decisions based on the above-mentioned processes are often biased by an abnormal sensitivity to positive and negative reinforcement, which results in abnormal sensitivity to performance feedback, skewed interpretations of ambiguity, inflated expectations, and asymmetrical belief updating. We have defined this abnormal cognitive processing as reinforcement-based cognitive biases (RBCBs). While the investigation of cognitive biases has a long history in economics and psychology, RBCBs in the context of AUD have been much less systematically investigated and, perhaps, neglected. This is surprising, as most of the decisions regarding alcohol-related behaviours are related to their positive and negative reinforcing effects. Although the causal role of the RBCBs in AUD has been emphasized by various psychological theories and investigating the interrelations between biased cognition and AUD could become a thriving area of research that capitalizes on the newest advances in cognitive neuroscience, psychology, and psychiatry, studying the nature and the extent to which RBCBs play a role in the development and maintenance of alcohol addiction still faces formidable methodological obstacles and does not provide definite answers regarding underlying mechanisms. 
In this review, we first briefly describe psychological and cognitive theories that implicated various aspects of reinforcement-biased cognition in the development, maintenance, and recurrence of alcohol addiction. Furthermore, in separate sections, we describe recent studies investigating the RBCBs, their neural, neurochemical, and pharmacological correlates, and possible interactions between RBCBs and trajectories of AUD. Finally, we describe how recent translational studies using state-of-the-art animal models could facilitate our understanding of the role of reinforcement sensitivity and RBCBs in various aspects of AUD.

\section{Psychological and cognitive theories and experimental evidence that implicated various aspects of reinforcement-biased cognition in the development, maintenance, and recurrence of alcohol addiction}

Several psychological theories sought to describe reinforcement-biased processes that are implicated in the development and progression of AUD (Skinner \& Aubin, 2010). According to the allostatic model (Koob, 2003; Koob \& Volkow, 2010), the early stages of alcohol addiction are primarily driven by positive reinforcement, which generally refers to the pleasant feelings and social enhancement effects that alcohol provides, while the later and more severe stages are driven by negative reinforcement, which encompasses relief from negative affective states such as stress or anxiety. The second model, the incentive-sensitization theory (Robinson \& Berridge, 1993), highlighted increasing subjective 'wanting' for alcohol that is driven by hypersensitization of the dopaminergic system, while a related theory postulated by Field \& Cox (2008) proposed that sensitization of the dopaminergic system drives alcohol attentional biases related to alcohol-related cues, leading to an increased craving. Finally, according to Cox \& Klinger (2011), some individuals drink alcohol for its positive reinforcing properties to increase their positive affective experience (e.g., mood enhancement), while others drink alcohol for its negative reinforcing properties to dampen their negative emotions and cope with distress and anxiety (tension reduction). The former, whose drinking is maintained by the rewarding effects of alcohol, are called 'reward drinkers', while the latter are called 'relief drinkers' (Ooteman, Koeter, Verheul, Schippers \& Van den Brink, 2006). Based on accumulating experimental evidence, it seems that there may be distinct types of AUD in which alcohol abuse is differentially driven by various mixtures of the above-mentioned reinforcement-based processes.

Individual differences in vulnerability to substance use disorders, including AUD, may be explained in light of reinforcement sensitivity theory (Corr, 2004). According to this hypothesis, every behaviour is governed by two conceptual brain systems: the behavioural approach system (BAS) and the behavioural inhibition system (BIS). These two systems differentially respond to the rewarding and punishing effects of alcohol. The BAS underlies approach motivation, positive affect, and reward learning processes and may contribute to the acquisition of AUD, while the BIS inhibits behaviour in response to stimuli signalling the loss of expected reward, uncertainty, and goal conflict and may support the maintenance of AUD (Corr, 2008). The BIS also gives rise to emotional distress and has been associated with trait negative affect, especially anxiety (McNaughton \& Gray, 2000). Research has suggested that the BAS can be further subdivided into three different types of reward sensitivity: (a) reward drive, relating to persistent pursuit of desired goals; (b) funseeking, relating to simple biological reinforcers that do not require planning; and (c) reward responsiveness, encompassing responses related to receiving or anticipating reward (Carver \& White, 1994; Corr, 2008). As alcohol consumption represents a high reward value (Everitt \& Robbins, 2005), it is not surprising that several studies demonstrated a relationship between positive reinforcement sensitivity, particularly in relation to fun-seeking, and higher alcohol intake, particularly in the form of binge drinking (Feil \& Hasking, 2008; Franken \& Muris, 2006; Loxton \& Dawe, 2001; O'Connor \& Paley, 2009; Voigt et al., 2009). It was also found that BAS sensitivity was related to both desire and negatively reinforcing aspects of alcohol craving. Subjects with high BAS sensitivity scores experienced significantly stronger desires and intention to drink alcohol and negative reinforcement craving during exposure to alcohol-related cues than subjects with low BAS sensitivity scores (Franken, 2002). Furthermore, persons with high BAS sensitivity experienced high negative reinforcement craving during this exposure (Franken, 2002). The relationship between BIS sensitivity and alcohol consumption is less clear. Although several investigators reported null associations between BIS and AUD (Hundt, Kimbrel, Mitchell \& Nelson-Gray, 2008; Kambouropoulos \& Staiger, 2001; O'Connor \& Colder, 2005), other studies suggested that the BIS, instead of inhibiting behaviour, draws 
attention to the potential dangers of a situation and functions as a conflict resolution system (Corr, 2008)). As a result, a more sensitive BIS leads to high anxiety, which could be reduced by indulging in drinking (Hasking, 2006). Indeed, it has been explicitly proposed that a sensitive BIS acts anxiogenically in response to health information, which may motivate protective health-related behaviours (Norman, Boer \& Seydel, 2005). By the same token, a more sensitive BIS may act as a protective factor due to the avoidance of potentially risky situations or aversive consequences (e.g., hangovers). This theory has been supported by several other studies (Kimbrel, Nelson-Gray \& Mitchell, 2007; O'Connor, Stewart \& Watt, 2009; Pardo, Aguilar, Molinuevo \& Torrubia, 2007) who found a negative correlation between the sensitivity of the BIS and the frequency and quantity of alcohol consumption. Similar results were reported by Knyazev and collaborators (2004), who found that a more sensitive BIS protects against substance abuse among youths. Taken together, it seems that the effects of BAS and BIS on the trajectories of alcohol addiction might be interactive (Figure 1). Indeed, several experiments demonstrated that high BAS sensitivity coupled with low BIS sensitivity was associated with significantly increased alcohol use (Kellough, Beevers, Ellis \& Wells, 2008; Wardell, O'Connor, Read \& Colder, 2011). Moreover, Wardell and colleagues (2011) demonstrated high BIS sensitivity as a risk factor for subsequent problematic drinking, but only when combined with high BAS sensitivity. When the sensitivity of BAS was low, BIS sensitivity was demonstrated to be protective against subsequent drinking. The above-mentioned studies suggested that the relationship between BIS and problematic drinking is moderated by BAS: in the absence of a sensitive BAS to shift attention towards the rewarding, tension-reducing properties of alcohol, the aversive consequences of drinking are more salient, leading to alcohol avoidance (Wardell, O'Connor, Read \& Colder, 2011).

The objective investigation of BAS and BIS sensitivity in people abusing alcohol can be conducted using a framework of neurophysiological and neuropsychological measures. The neurophysiological measures are mostly based on the peripheral assessment of cardiac and electrodermal reactivity during appetitive responding for reward and during extinction of appetitive responding (i.e., frustrative non-reward), respectively (Beauchaine, 2001; Fowles, 1988; Iaboni, Douglas \& Ditto, 1997; Tranel, 1983). In turn, the neuropsychological evaluation is usually conducted using self-report questionnaires that assess individual differences in BAS and BIS sensitivity by the degree to which respondents endorse prototypical approach- and avoidance-related behaviours. This strategy is exemplified in the BIS/BAS scales of Carver and White (1994), which were developed with the explicit purpose of assessing individual differences in state reactivity of these systems. Recently, investigators have developed computerized neuropsychological tests to experimentally measure sensitivity to reinforcement (Cools, Clark, Owen \& Robbins, 2002; Evers et al., 2005; Paulus, Hozack, Frank \& Brown, 2002; Paulus, Hozack, Frank, Brown \& Schuckit, 2003; Slaney, Hinchcliffe \& Robinson, 2018). These tests offer many advantages over conventional pencil-and-paper testing because they standardize aspects of administration and automate data collection and analysis. One of these tests is the probabilistic reversal learning (PRL) test, offering an effective way of measuring an individual's sensitivity to reinforcement by assessing win-stay and lose-shift behaviours (WSLS) following rewarding and punishing feedback, respectively. In this paradigm, subjects are presented with two (sometimes more) stimuli on each trial and using trial-and-error feedback after each response, learn to select the stimulus that is usually correct (rewarded on a majority or punished on a minority of trials) and to avoid the stimulus that is usually incorrect (punished on a majority or rewarded on a minority of trials). This rule intermittently reverses such that the stimulus that was usually rewarded becomes usually punished/unrewarded and vice versa. Consequently, responding must be adjusted to gain the reward and avoid punishment. Rewarded outcomes followed by a decision to stay with the response that delivered them (win-stays) constitute a measure of sensitivity to positive reinforcement. Conversely, lose-shift ratios, calculated by dividing punishing outcomes after which the subject changed the choice by the total number of punished trials on a given stimulus, represent a measure of sensitivity to negative reinforcement. The use of probabilistic reinforcement increases the complexity of the task in such a way that the information from any given choice is insufficient to guide future behaviour, and subjects must engage cognitive functions to track the reward and punishment history for both stimuli to determine the stimulus that is more beneficial overall. The PRL paradigm has been recently successfully applied in a number of studies used to investigate the neuroanatomical and neurochemical correlates of reinforcement sensitivity in humans and non-human animals (Rygula, Noworyta-Sokolowska, Drozd \& Kozub, 
2018). These will be discussed in subsequent sections of this paper.

Individual differences in reinforcement sensitivity may influence the acquisition and maintenance of positively and negatively biased alcohol-drinking outcome expectancies, which can be defined as positively or negatively inflated beliefs about the effects of alcohol on behaviour, cognition, moods, and emotions (Leigh, 1989). These expectations, in turn, have a pivotal role in determining decisions about alcohol drinking and trajectories of its use (Jones, Corbin \& Fromme, 2001). Beginning with the seminal work of Brown and her colleagues (Brown, Goldman, Inn \& Anderson, 1980; Goldman, 1994), many studies have documented positively biased outcome expectancies for engaging in alcohol drinking, together with minimized negative expectancies and poor selfefficacy or beliefs about one's ability to cope without alcohol, can maintain addictive behaviours and predict relapse (Brown, Christiansen \& Goldman, 1987; Christiansen \& Goldman, 1983; Floyd \& Widaman, 1995; Fromme \& D'Amico, 2000; Fromme, Stroot \& Kaplan, 1993). In these experiments, alcohol-drinking outcome expectancies were measured using structured interviews of focus groups and the self-report questionnaires in which individuals endorse each questionnaire item as to whether or not they hold that particular expectancy. A sum of the expectancy endorsements representing the individual's overall alcohol outcome expectancy is further positively associated with alcohol consumption, e.g., subjects who drank in a frequent, social manner, expected alcohol to enhance their social behaviour (Christiansen \& Goldman, 1983; Lee, Maggs, Neighbors \& Patrick, 2011). Other studies have investigated the potential associations of different categories of alcohol outcome expectancies with subsequent drinking behaviour. A study by Leeman and collaborators (2009) found significant associations of euphoria and social enhancement expectancies with binge alcohol use, while the study by Pabst and colleagues (2010) found the same association concerning sexual and social relationship enhancement expectancies. Other cross-sectional and longitudinal studies have demonstrated that greater numbers of positive alcohol outcome expectancies are associated with greater numbers of negative consequences independent of the level of consumption (Blume \& Blume, 2014; Blume, Lostutter, Schmaling \& Marlatt, 2003).

Abnormal perceptions of risks associated with alcohol-drinking outcomes can also be considered within the concept of unrealistic optimism bias (Sharot, Korn \& Dolan, 2011), which encompasses two different phenomena: unrealistic comparative optimism and unrealistic absolute optimism (Shepperd, Klein, Waters \& Weinstein, 2013). The former refers to people's tendency to view the risks as lower for themselves than for the others, while the latter refers to unrealistically positive risk assessment when compared to an objective criterion, such as an actual risk assessment. The mechanism that allows individuals to maintain or arrive at unrealistically positive beliefs in the face of disconfirming evidence was described for the first time by Sharot, Korn and Dolan (2011). The results of this study demonstrated that when people update their initial risk estimates, they tend to incorporate desirable information (i.e., information that risks are lower than expected) to a greater extent than undesirable information (i.e., information that risks are higher than expected). In those with AUD, the asymmetric reliance on information about alcohol-drinking outcomes, dependent on their valence, may result in larger updates after desirable/positive information than after undesirable/negative information and in this way affect drinking trajectories. When considering expectancies and beliefs about the effects of alcohol, it is also important to mention the interpretation of ambiguity in drinking outcomes. Indeed, positive/appetitive alcohol-related expectations are often associated with more negative/inhibitory information arising from memories. This conflict and the bias in its interpretation can be assessed using various types of ambiguous-cue interpretation (ACI) paradigms. In this experimental and fully translational approach, participants initially learn to discriminate two stimuli (e.g., tones of different frequencies), which acquire emotional and motivational value due to subsequent feedback (monetary gain or avoidance of monetary loss). After such an acquisition phase, the test phase introduces ambiguous stimuli (e.g., tones of intermediate frequencies) that serve as a measure of interpretation bias since the response to these stimuli indicates the participants' expectation of rewarding or potentially punishing effects of their decision (Papciak \& Rygula, 2017; Schick, Wessa, Vollmayr, Kuehner \& Kanske, 2013). Other ways of assessing biased interpretations of ambiguity include using single ambiguous words individually presented to the participant, which are typically either homophones (words that sound the same yet have different spellings and meanings, e.g., meat/meet or sea/see) or homographs (words with identical spelling yet distinct mean- 
ings, e.g., bat - animal or wooden club; change - to alter or money) (Drury, 1969; Gorfein \& Weingartner, 2008). Homophones and homographs used in these studies usually have both threatening/emotional and neutral interpretations, and an interpretation bias is established as the number or proportion of each type of interpretation made by participants from a list of such words (Hindash \& Amir, 2012; Warren, Warren, Green \& Bresnick, 1978). Ambiguity interpretation biases can also be investigated using ambiguous images, often with emotional and neutral faces (Beevers, Wells, Ellis \& Fischer, 2009; Schaefer, Baumann, Rich, Luckenbaugh \& Zarate Jr, 2010).

Another conceptual approach focuses not on expectancies regarding alcohol-drinking outcomes per se but on the mechanisms that underlie them. These mechanisms have been defined as attributional styles, which Alloy and colleagues (1984) described as the manner in which a person explains the causes of prior outcomes. Indeed, following positive or negative experiences with alcohol, people often wonder why the event occurred (DeJoy, 1989; Jessup et al., 2014). To answer this question, they make causal attributions based upon different dimensions: internal-external (whether the event was caused by themselves (internal) or by the situation (external)), stable-unstable (whether the cause of an experience is constant and likely to happen again (stable) or pliable and unlikely to reoccur (unstable)), and global-specific (whether the cause may affect many areas of one's life (global) or only one area (specific)) (Weiner, 1985). From this perspective, attributing negative outcomes to causes that are internal to the person, stable and global can reflect pessimism, learned helplessness, and addiction vulnerability (Scheier \& Carver, 2018). Following the above-mentioned framework, Weiner (1985) and Marlatt \& Donovan (2005) proposed that a certain pattern of attribution promotes relapse in substance use. They argued that a combination of internal, stable, and global attributions contributes to maladaptive thought patterns concerning addiction and promotes relapse. This pattern, often experienced by an individual after a return to substance use following a period of self-imposed abstinence from substances, has been named the abstinence violation effect (Curry, Marlatt \& Gordon, 1987). Within this model, bleak attributions regarding future reactions to alcohol exposure lead individuals to believe that fighting addiction is pointless and, in turn, to relapse rather than returning to abstinence (Walton, Castro \& Barrington, 1994). This conceptual orientation has given rise to assessment techniques that focus on measuring the causes that people identify for the outcomes that they experience. Typically, respondents are asked to imagine being in outcome scenarios and to indicate how they would explain the described outcomes (Peterson, Semmel, Von Baeyer, Abramson, Metalsky \& Seligman, 1982) or to analyse naturally occurring verbatim materials (e.g., newspaper articles or speeches) for explanatory style (Schulman, Castellon \& Seligman, 1989).

Taken together, these studies consistently support the notion that various types of RBCBs, such as abnormal sensitivity to performance feedback, biased interpretation of ambiguity, inflated expectations, and asymmetrical belief updating, can be differentially associated with drinking at different stages of AUD (Figure 2). Understanding the underlying mechanisms could provide useful information for tailoring prevention and intervention efforts. For example, medications used in the early stages of addiction should target positive reinforcement sensitivity, while treatments that target alcohol-dependent individuals in the late stages of addiction may need to simultaneously target positive and negative reinforcement processes. In other words, understanding individuals' positive and negative reinforcement profiles and resulting cognitive biases can provide important information for personalized medicine. Indeed, there is preliminary evidence for the effectiveness of tailoring pharmacotherapies to AUD patients based on their positive and negative reinforcement tendencies (Mann et al., 2018; Roos, Mann \& Witkiewitz, 2017). For example, an opioid receptor antagonist,

naltrexone, has been found to be particularly effective for reward drinkers, while acamprosate, which has been shown to down-regulate the glutamatergic system, is particularly effective for relief drinkers (Roos, Mann \& Witkiewitz, 2017). Specific neurochemical correlates of the interactions between RBCBs and AUD, as well as potential future treatment targets, are described in the next sections.

Neurochemical correlates of reinforcement-based cognitive biases as potential targets for pharmacological treatment of alcohol addiction

Influential neurocomputational models emphasize dopamine (DA) as a neurochemical correlate of reinforce- 
ment learning (Bromberg-Martin, Matsumoto \& Hikosaka, 2010; Eisenegger et al., 2014; Frank, Seeberger \& O'Reilly R, 2004; Samson, Frank \& Fellous, 2010). Indeed, the role of DA in the mediation of positive reinforcement and resulting cognitive biases is hard to overestimate. In a classic study, Frank and colleagues (2004) demonstrated that lowered availability of DA (as in Parkinson's patients off medication) was associated with worse learning from the positive than from the negative outcomes. Importantly, this effect could be reversed by pharmacological boosting of dopaminergic neurotransmission. As suggested by Frank (2005), the opposite effects of low and high DA availability reflected DA-induced shifts in the balance between the BAS/Go/direct and BIS/NoGo/indirect pathways of the basal ganglia with low DA shifting the balance towards the NoGo pathway (impairing learning from positive feedback/reward relative to learning from punishment) and high DA shifting the balance towards the Go pathway (improving learning from positive feedback/reward relative to learning from punishment). Several other studies reported impairments in learning from punishment by dopaminergic therapy (Bodi et al., 2009; Cools, Barker, Sahakian \& Robbins, 2001; Cools, Frank, Gibbs, Miyakawa, Jagust \& D'Esposito, 2009; Moustafa, Cohen, Sherman \& Frank, 2008; Swainson, Rogers, Sahakian, Summers, Polkey \& Robbins, 2000). In the study by Pessiglione and colleagues (2006), enhancement of DA activity by administration of L-3,4-dihydroxyphenylalanine (L-DOPA) increased the frequency with which subjects chose high-probability gain but not the frequency with which they chose low-probability loss. It has been proposed that pharmacological boosting of DA neurotransmission increases tonic levels of DA within the striatum, which in turn occludes DA dips, which signal negative feedback, differentially affecting reward versus punishment-based learning (Frank, 2005; Grace \& Rosenkranz, 2002). The role of DA in regulating feedback sensitivity has been recently confirmed in a preclinical study by Grospe and collaborators (2018). In that study, 6-hydroxydopamine-induced dopaminergic lesions within the rat dorsomedial striatum resulted in significantly increased negative reinforcement sensitivity. Notably, the sensitivity to reinforcement and associated cognitive biases also seem to be regulated at the receptor level. The important role of dopamine $\mathrm{D}_{2}$ receptor gene polymorphism (DRD2-TAQ-IA) in reinforcement learning has been demonstrated by Klein and colleagues (2007) using a neuroimaging paradigm. In this study, in a probabilistic learning task, $\mathrm{A} 1$ allele carriers with reduced dopamine $\mathrm{D}_{2}$ receptor densities learned to avoid actions with negative consequences less efficiently than those without it. A1 subjects have also been shown to be impaired in the ability to sustain a newly rewarded response after a reversal and demonstrated a generally decreased tendency to stick with a rewarded response (Jocham, Klein, Neumann, von Cramon, Reuter \& Ullsperger, 2009). In 2015, Cox and collaborators (2015), using positron emission tomography (PET) with two selective DA receptor radioligands ([11C]SCH23390 and [11C]raclopride), demonstrated that individual differences in dopaminergic $\mathrm{D}_{1}$ and $\mathrm{D}_{2}$ receptor binding determine the effectiveness of learning from positive and negative reinforcement, respectively. Studies in humans were complemented by a series of elegant studies using animal models. A study by Groman and collaborators (2016), using PET in rats performing a three-choice spatial PRL task, demonstrated a role for dopamine $\mathrm{D}_{3}$ receptors in reinforcement learning. In that study, greater midbrain dopamine $\mathrm{D}_{3}$ receptor availability (indicated by [11C]-(+)-PHNO binding) was associated with a lower sensitivity to positive reinforcement, resulting in a lower rate of learning. The role of dopamine $\mathrm{D}_{3}$ receptors in sensitivity to reinforcement was further confirmed following administration of a dopamine $\mathrm{D}_{3} \backslash$ sout-receptor agonist, pramipexole, which impaired the performance of rats in a very similar way (Groman et al., 2016). These results suggest that in addition to dopamine $\mathrm{D}_{1} / \mathrm{D}_{2}$, dopamine $\mathrm{D}_{3}$ receptor dysregulation may also underlie abnormal reinforcement sensitivity, and they implicate that these receptors may be a novel target for AUD treatment. In, Sharot and colleagues demonstrated that administration of LDOPA during the imaginative construction of positive future life events subsequently enhanced estimates of the hedonic pleasure to be derived from these same events (Sharot, Shiner, Brown, Fan \& Dolan, 2009). This study was supported by a report in 2012 (Sharot, Guitart-Masip, Korn, Chowdhury \& Dolan, 2012), which revealed that administration of L-DOPA increased optimism bias by impairing the ability to update beliefs in response to undesirable information about the future. The latter converged with the above-mentioned observations from patients with Parkinson's disease, where enhanced DA levels led to impaired learning from unwanted outcomes (Frank, Seeberger \& O'Reilly R, 2004). These findings provided evidence that DA modulates subjective hedonic expectations and impacts belief formation by reducing negative expectations regarding the future. 
Along with DA, serotonin (5-HT) is the second neurotransmitter crucially implicated in reinforcement learning. Published reports (Bari et al., 2010; Chamberlain, Muller, Blackwell, Clark, Robbins \& Sahakian, 2006; Cools, Robinson \& Sahakian, 2008; Fischer \& Ullsperger, 2017; Rygula et al., 2015; Sachs, Rodriguiz, Tran, Iyer, Wetsel \& Caron, 2015) have suggested that increasing 5-HT neurotransmission leads to a reduced sensitivity to aversive outcomes, whereas reducing 5-HT transmission, by way of either acute tryptophan depletion (ATD), a procedure that has been used extensively to study the effect of low 5-HT levels in the human brain, pre-synaptic receptor stimulation (acting to temporarily down-regulate 5-HT transmission), or up-regulation of the serotonin transporter (SERT), leads to an increased sensitivity to aversive outcomes. Indeed, a study by Chamberlain and colleagues (2006) demonstrated that a low, acute dose of the selective serotonin reuptake inhibitor (SSRI) citalopram, which has been postulated to affect pre-synaptic serotonin $5-\mathrm{HT}_{1 \mathrm{~A}}$ autoreceptors, increases the sensitivity to negative feedback in the PRL task. Similar effects were reported in a study by Cools and collaborators (2008), where ATD enhanced the ability of subjects to predict punishment without affecting reward predictions. The results of the above-mentioned studies were complemented by the report by den Ouden and collaborators (2013), who studied the role of 5-HT (and DA) in reinforcement sensitivity as a function of two polymorphisms in the genes encoding the 5-HT and DA transporters (SERT: 5HTTLPR plus rs25531; DAT1 3'UTR VNTR). The results of this study revealed that allelic variation in SERT predicted behavioural adaptation following punishment. Specifically, L' homozygosity, which has been linked with increased SERT binding and decreased levels of extracellular 5-HT (Willeit \& Praschak-Rieder, 2010), was associated with increased negative reinforcement learning (den Ouden et al., 2013). The role of serotonin in RBCBs has been supported by ample evidence from research using animal models. In 2010, Bari and collaborators (2010) demonstrated that different manipulations of 5-HT neurotransmission in rats resulted in altered sensitivity to positive and negative reinforcement. In this study, acute administration of a high dose of SSRI citalopram decreased negative feedback sensitivity by lowering the ratio of lose-shift behaviours. In contrast, acute administration of a low dose of this drug, which was postulated to temporarily silence 5-HT system activity via inhibitory serotonin 5 - $\mathrm{HT}_{1 \mathrm{~A}}$ autoreceptor activation in the raphe nuclei, similar to the above-mentioned study by Chamberlain and collaborators (2006), increased sensitivity to negative reinforcement. A similar effect was reported following global 5-HT depletion (Bari et al., 2010). In 2012, Ineichen and colleagues (2012) demonstrated in an automated two-choice operant spatial discrimination paradigm that genetic reduction in SERT function investigated in heterozygous mutant mice from a SERT null mutant strain led to a decreased sensitivity to negative feedback, which was an effect similar to that observed by Bari and collaborators (2010) following acute administration of the higher dose of citalopram. Both of the mentioned manipulations also caused increased ratios of win-stay behaviours, indicating increased sensitivity to positive reinforcement (Ineichen et al., 2012), an effect confirmed recently by Wilkinson and colleagues (2020). The results of the study by Rygula and collaborators (2014) revealed the important role of 5-HT in modulating cognitive judgement bias in rats. In that study, the SSRI citalopram at a low dose of $1 \mathrm{mg} / \mathrm{kg}$ significantly biased animals towards positive interpretation of the ambiguous cues, while at higher doses $(5$ and $10 \mathrm{mg} / \mathrm{kg}$ ), the animals interpreted the ambiguous cues more negatively. Interestingly, a study from 2017 further demonstrated that the effects of acute 5-HT manipulations on the interpretation of ambiguity might depend on the basal valence of cognitive judgement bias (Golebiowska \& Rygula, 2017). In that study, acute administration of escitalopram caused a 'pessimistic' shift in the interpretation of ambiguous cues in animals classified as 'optimistic' and had no significant effects on those previously classified as 'pessimistic'.

Taken together, published reports suggest that increasing 5-HT transmission leads to a reduced sensitivity to aversive outcomes, whereas reducing 5-HT transmission, by way of either ATD, pre-synaptic receptor stimulation, or up-regulation of SERT, leads to an increased sensitivity to aversive outcomes. These results strongly suggest 5-HT as a potential target and serotonergic manipulations as effective treatment strategies in modulating alcohol-drinking outcome expectations.

To summarize this section, psychopharmacologic manipulation of DA and 5-HT neurotransmitter systems implicated in RBCBs may have the potential to provide insights into the development and maintenance of alcohol addiction and should be considered as targets for pharmacological treatment of AUD (Figure 3). Of 
particular interest is the potential to experimentally vary reinforcement sensitivity and outcome expectations and to do so in a way that builds a direct paradigmatic bridge with the relevant alcohol literature. Further challenges include identification of the specific interactions between neurochemical correlates of RBCBs and brain processes involved in alcohol addiction.

\section{Preclinical and translational studies using state-of-the-art animal models that are useful for elucidating the role of RBCBs in various aspects of AUD and its treatment}

Creating preclinical models is essential for elucidating the reinforcement-based cognitive mechanisms that contribute to addiction-related behaviours. However, the main difficulty with modelling RBCBs using nonhuman animals is capturing these inherently complex processes using relatively simple behavioural protocols. One of the most promising preclinical tools that can be used for the investigation of biased sensitivity to reinforcement in animal models is mentioned in the previous sections, the PRL paradigm (Figure 4). In the rat version of the PRL task, the animals are trained to press levers (Rychlik, Bollen \& Rygula, 2017; Rygula \& Popik, 2016) or nose-poke holes (Bari et al., 2010) in an operant conditioning chamber to receive a food reward. Choosing one of them (the 'correct' lever or hole) is associated with a high probability of receiving a reward (a drop of sucrose solution or reward pellet) and a low probability of receiving a punishment (mild electric foot shock or lack of reward and time out). Conversely, choosing the other one (the 'incorrect' lever or hole) is associated with a high probability of receiving the punishment and only a small chance of receiving the reward. The probabilities of receiving the reward and punishment upon choosing the 'correct' lever or hole are usually set to $80 \%$ and $20 \%$, respectively, and vice versa for the 'incorrect' lever or hole. The animals have to adjust their behaviour by responding to the appropriate levers or holes to maximize reward and minimize punishment delivery while disregarding occasional misleading positive or negative feedback. After several consecutive choices of the 'correct' lever or hole, the reversal criterion is reached, and the probabilities reverse; that is, the previously 'correct' lever or hole becomes 'incorrect', and vice versa. Similar to humans, to assess the sensitivity of the animals to positive and negative reinforcement, behaviour is analysed according to the outcome of each preceding trial and based on the WSLS analysis, where the proportion of win-stay behaviours indexes sensitivity to positive reinforcement while the proportion of lose-shifts indicates sensitivity to negative reinforcement. In the aforementioned landmark study, using a PRL task analogous to that used in humans, Bari and colleagues (2010) demonstrated that different manipulations of 5 -HT neurotransmission in rats resulted in significant changes in reinforcement sensitivity. These results were further complemented by a study in a non-human primate, the marmoset, employing a preclinical and translational version of the PRL task, which demonstrated that 5-HT depletions within the orbitofrontal cortex and amygdala manifested impairments in overall reinforcement sensitivity rates, including temporal learning from both rewarding and negative outcomes (Rygula et al., 2015). A study by Rychlik and collaborators (2017) demonstrated, using a preclinical version of the PRL paradigm, that the non-monoaminergic compound ketamine selectively down-regulates sensitivity to negative outcomes in animals. In this study, acute treatment with ketamine significantly and persistently decreased the ratio of lose-shift behaviours in a manner similar to that observed following the administration of higher doses of the SSRI citalopram (Bari et al., 2010). These results were recently supported by a study by Wilkinson and collaborators (2020), who also observed an interaction between ketamine treatment and feedback type with a trend towards decreased negative feedback sensitivity in the preclinical PRL paradigm. Ketamine was also reported to have robust positive effects on the interpretation of ambiguous cues (Hales, Houghton \& Robinson, 2017). Given the similarity in the effects of citalopram and ketamine, it was hypothesized that the effects of the latter were mediated indirectly by 5-HT neurotransmission (Rychlik, Bollen \& Rygula, 2017). Indeed, recent reports have demonstrated that in addition to affecting glutamatergic neurotransmission via $\mathrm{N}$-methyl-D-aspartate receptors (NMDARs), ketamine also potentiates 5 -HT release in the prefrontal cortex via amino-3-hydroxy-5-methyl-4-isoxazolepropionic acid receptors (AMPARs) in the raphe nucleus (Nishitani et al., 2014). As demonstrated by recent studies (Drozd, Rychlik, Fijalkowska \& Rygula, 2019; Noworyta \& Rygula, 2021; Rygula \& Popik, 2016), the preclinical version of the PRL task may be used not only for investigating neuroanatomical or neurochemical bases of reinforcement sensitivity but also for evaluating how this sensitivity interacts with other cognitive processes and pharmacological treatment outcomes. In a study 
by Rygula and Popik (2016), the PRL task was employed to investigate how 'optimistic' and 'pessimistic' animals incorporated feedback (both rewarding and punishing) in their decisions in a changing and uncertain environment. The results of this study demonstrated the interrelation and co-existence of two cognitive biases (the rats classified as 'pessimistic' were significantly more sensitive to negative feedback than their 'optimistic' conspecifics) that may predict vulnerability to various psychiatric disorders, including AUD. A study by Noworyta-Sokolowska and colleagues (2019) demonstrated for the first time in rodents that sensitivity to negative and positive reinforcement could be considered a stable and enduring behavioural trait. It also showed that these traits were independent of each other and that trait sensitivity to positive reinforcement is associated with cognitive flexibility. These results have been supported by computational modelling (Noworyta-Sokolowska, Kozub, Jablonska, Rodriguez Parkitna, Drozd \& Rygula, 2019). Several preclinical studies in rats have investigated the neuroanatomical background of biased sensitivity to reinforcement. In 2014, Dalton and colleagues (2014), using a preclinical version of the PRL paradigm, found that the nucleus accumbens shell and core facilitate reward-seeking in a distinct yet complementary manner, with the shell guiding response selection to the actions more likely to yield the reward and the core simply promoting the approach towards reward-associated stimuli. In 2016, the same group demonstrated that inactivation of the medial orbitofrontal cortex rendered animals less sensitive to either positive or negative feedback, while lateral orbitofrontal cortex activity was implicated in behaviours following violations of reward expectancies signalled by negative feedback (Dalton, Wang, Phillips \& Floresco, 2016). In the same study, inactivation of the prelimbic cortex increased sensitivity to positive feedback and reduced sensitivity to negative feedback. Since the animals tended to select the recently rewarded choice more often, regardless of whether the previous choice was correct or not, this surprising effect has been proposed to result from a form of 'reward myopia' (Dalton, Wang, Phillips \& Floresco, 2016). In 2019, Drozd and colleagues (2019) reported the reinforcement modulating effects of the antidepressant drugs mirtazapine and agomelatine in the PRL task, and these results were supported a year later in a study by Wilkinson and collaborators (2020). Recent research using a preclinical version of the PRL paradigm identified 4 phenotypes of sensitivity to negative and positive reinforcement in rats and reported statistically significant differences between the investigated phenotypes in the effects of acute treatment with SSRI escitalopram on anxiety (Noworyta \& Rygula, 2021). These results demonstrated that trait sensitivity to reinforcement could have important implications for the effectiveness of pharmacological interventions, including in AUD. Last but not least, as mentioned above, the fear of negative outcomes has a powerful influence on decisions regarding drinking. A study by Clarke and colleagues (2014) in marmoset monkeys demonstrated, using an approach-avoidance conflict task, that inactivation of anterior orbitofrontal or ventrolateral prefrontal cortices increased general negative bias in decision making via two distinct cognitive mechanisms - elevated uncertainty and attentional disruption, respectively. The differentiation of the component neural mechanisms underlying punishment processing revealed in that study provided important insight into the efficacy of cognitive-behavioural therapy in AUD, which may be more successful in a patient poor at predicting than in one deficient in attentional control.

\section{Conclusions and future directions}

RBCBs occur in many neuropsychiatric disorders and may even be helpful in defining them. Over the last decade, the consideration of RBCBs as important factors in the development of alcohol addiction has enriched and strengthened the field of inquiry regarding AUD and opened new avenues of research. To further define the relationships between RBCBs and AUD, in this review, we have advocated a psychological approach based largely on cognitive and learning theories, which combined with neural and neurochemical analyses may facilitate our understanding of this disease. We have also highlighted the development of translational tools, which herald a new phase of research in this field, that will more readily make contact between the clinic and basic neuroscience. We have also described the effects of pharmacological manipulations, as a multi-level analysis of RBCBs may identify medications that can be used for different expressions of what may, in fact, be common disorders resulting from reinforcement biased cognition. The possible 're-purposing' of agents such as SSRIs and ketamine may provide an initial impetus for this enterprise. Finally, we also mentioned the concept of endophenotypes, as a focus on individual differences in this diverse disorder may inform genetic approaches to understanding its aetiology. Based on available knowledge, it seems that using 
rodent-based models, such as the preclinical PRL or ACI, can help to reveal neurobiological processes that modulate RBCBs and their role in AUD. This in turn may help to reveal novel drug targets for improved and personalized treatments. Although we hope this review has provided enough evidence to support the validity of the claim that RBCBs can determine the trajectories of alcohol addiction, there are still a number of outstanding issues that future research will need to address. First, we still do not know the degree of the causal relationship between increased/decreased sensitivity to negative/positive reinforcement as a trait and vulnerability to AUD. The development of translational preclinical tests of RBCBs should help to elucidate this issue and may help to design personalized treatments based on these cognitive variables. Second, although we know that there are distributed changes in neural activity within multiple regions of the PFC and the striatum associated with altered activity of serotonergic and dopaminergic systems that occur over the course of AUD and can persist into periods of abstinence, unfortunately, studies looking at neurochemical correlates of altered reinforcement sensitivity in this context are still limited, and further investigation in this area is needed. We also still need more detailed pharmacological studies using drugs with known profiles in humans to understand the value of targeting RBCBs in AUD. It will be highly desirable to use voltammetry, optogenetics, or other biosensors and electrophysiological measures to characterize neuronal pathways and to elucidate the exact function and dynamic balance between cortical and subcortical regions involved in RBCBs and AUD. Finally, further conceptual and empirical development is required to provide an integrated account of the role of reinforcement sensitivity in the aetiology, development, and recurrence of AUD.

\section{Acknowledgement}

This work was supported by the Polish National Science Centre (Research grant 2018/31/B/NZ7/03690 to RR) and by the statutory funds of the Maj Institute of Pharmacology Polish Academy of Sciences.

Figures

Figure 1. Sensitivity of the behavioural activation system (BAS) and the behavioural inhibition system (BIS) is associated with various reward- and punishment-driven processes. Abnormal sensitivity of these systems induces reinforcement-based cognitive biases (RBCBs) affecting the development and trajectory of alcohol use disorder (AUD) .

Figure 2. Reinforcement-based cognitive biases associated with the pathophysiology of alcohol use disorder (AUD) include abnormal sensitivity to positive and negative feedback, biased interpretation of ambiguity and conflict situations, lowered negative and inflated positive outcome expectations, and unrealistic optimism. Understanding the underlying mechanisms could provide useful information for tailoring prevention and intervention efforts.

Figure 3. Dopamine (DA) and serotonin (5-HT) modulate reinforcement-based cognitive biases. Several pharmacological manipulations (selective serotonin reuptake inhibitors (SSRIs) and L-3,4dihydroxyphenylalanine (L-DOPA)) have been demonstrated to modulate feedback sensitivity and outcome expectations in humans and animal models. Pharmacological targeting of these biases may help to shape the trajectories of alcohol use disorder (AUD).

Figure 4. The probabilistic reversal learning (PRL) paradigm. One of the most promising, translational tools that can be used for the investigation of biased sensitivity to reinforcement in animal models and in humans.

\section{References}

Alloy LB, Peterson C, Abramson LY, \& Seligman ME (1984). Attributional style and the generality of learned helplessness. Journal of personality and social psychology 46: 681.

Bari A, Theobald DE, Caprioli D, Mar AC, Aidoo-Micah A, Dalley JW, et al. (2010). Serotonin modulates sensitivity to reward and negative feedback in a probabilistic reversal learning task in rats. Neuropsychopharmacology 35: 1290-1301. 
Beauchaine T (2001). Vagal tone, development, and Gray's motivational theory: toward an integrated model of autonomic nervous system functioning in psychopathology. Dev Psychopathol 13: 183-214.

Beevers CG, Wells TT, Ellis AJ, \& Fischer K (2009). Identification of emotionally ambiguous interpersonal stimuli among dysphoric and nondysphoric individuals. Cognitive therapy and research 33:283-290.

Blume AW, \& Blume AK (2014). Alcohol outcome expectancies and consequences: Do people think themselves into and out of consequences. Psychology Applications \& Developments: 17-23.

Blume AW, Lostutter TW, Schmaling KB, \& Marlatt GA (2003). Beliefs about drinking behavior predict drinking consequences. J Psychoactive Drugs 35: 395-399.

Bodi N, Keri S, Nagy H, Moustafa A, Myers CE, Daw N, et al.(2009). Reward-learning and the noveltyseeking personality: a between- and within-subjects study of the effects of dopamine agonists on young Parkinson's patients. Brain 132: 2385-2395.

Bromberg-Martin ES, Matsumoto M, \& Hikosaka O (2010). Dopamine in motivational control: rewarding, aversive, and alerting. Neuron 68: 815-834.

Brown SA, Christiansen BA, \& Goldman MS (1987). The Alcohol Expectancy Questionnaire: an instrument for the assessment of adolescent and adult alcohol expectancies. Journal of studies on alcohol 48:483-491.

Brown SA, Goldman MS, Inn A, \& Anderson LR (1980). Expectations of reinforcement from alcohol: their domain and relation to drinking patterns. Journal of consulting and Clinical Psychology 48:419.

Carver CS, \& White TL (1994). Behavioral-Inhibition, Behavioral Activation, and Affective Responses to Impending Reward and Punishment - the Bis Bas Scales. Journal of Personality and Social Psychology 67: 319-333.

Chamberlain SR, Muller U, Blackwell AD, Clark L, Robbins TW, \& Sahakian BJ (2006). Neurochemical modulation of response inhibition and probabilistic learning in humans. Science 311: 861-863.

Christiansen BA, \& Goldman MS (1983). Alcohol-related expectancies versus demographic/background variables in the prediction of adolescent drinking. Journal of Consulting and Clinical Psychology 51:249-257.

Clarke HF, Cardinal RN, Rygula R, Hong YT, Fryer TD, Sawiak SJ, et al. (2014). Orbitofrontal dopamine depletion upregulates caudate dopamine and alters behavior via changes in reinforcement sensitivity. J Neurosci 34: 7663-7676.

Cools R, Barker RA, Sahakian BJ, \& Robbins TW (2001). Enhanced or impaired cognitive function in Parkinson's disease as a function of dopaminergic medication and task demands. Cereb Cortex 11:11361143.

Cools R, Clark L, Owen AM, \& Robbins TW (2002). Defining the neural mechanisms of probabilistic reversal learning using event-related functional magnetic resonance imaging. J Neurosci 22:4563-4567.

Cools R, Frank MJ, Gibbs SE, Miyakawa A, Jagust W, \& D'Esposito M (2009). Striatal dopamine predicts outcome-specific reversal learning and its sensitivity to dopaminergic drug administration. J Neurosci 29: 1538-1543.

Cools R, Roberts AC, \& Robbins TW (2008). Serotoninergic regulation of emotional and behavioural control processes. Trends Cogn Sci 12: 31-40.

Cools R, Robinson OJ, \& Sahakian B (2008). Acute tryptophan depletion in healthy volunteers enhances punishment prediction but does not affect reward prediction. Neuropsychopharmacology 33: 2291-2299.

Corr PJ (2004). Reinforcement sensitivity theory and personality. Neurosci Biobehav Rev 28: 317-332.

Corr PJ (2008). The reinforcement sensitivity theory of personality and psychopathology. Int J Psychophysiol 69: 151-152. 
Cox SM, Frank MJ, Larcher K, Fellows LK, Clark CA, Leyton M, et al. (2015). Striatal D1 and D2 signaling differentially predict learning from positive and negative outcomes. Neuroimage 109:95-101.

Cox WM, \& Klinger E (2011). A motivational model of alcohol use: Determinants of use and change. In Handbook of motivational counseling: Goal-based approaches to assessment and intervention with addiction and other problems, 2nd ed. Wiley Blackwell, pp 131-158.

Curry S, Marlatt GA, \& Gordon JR (1987). Abstinence violation effect: validation of an attributional construct with smoking cessation. Journal of Consulting and Clinical Psychology 55: 145.

Dalton GL, Phillips AG, \& Floresco SB (2014). Preferential involvement by nucleus accumbens shell in mediating probabilistic learning and reversal shifts. J Neurosci 34: 4618-4626.

Dalton GL, Wang NY, Phillips AG, \& Floresco SB (2016). Multifaceted Contributions by Different Regions of the Orbitofrontal and Medial Prefrontal Cortex to Probabilistic Reversal Learning. J Neurosci 36: 19962006.

DeJoy DM (1989). An Attribution Theory Perspective on Alcohol-Impaired Driving. Health Education Quarterly 16: 359-372.

den Ouden HE, Daw ND, Fernandez G, Elshout JA, Rijpkema M, Hoogman M, et al. (2013). Dissociable effects of dopamine and serotonin on reversal learning. Neuron 80: 1090-1100.

Drozd R, Rychlik M, Fijalkowska A, \& Rygula R (2019). Effects of cognitive judgement bias and acute antidepressant treatment on sensitivity to feedback and cognitive flexibility in the rat version of the probabilistic reversal-learning test. Behav Brain Res 359:619-629.

Drury DA (1969). Homographs and pseudo-homographs. Word Ways 2:10.

Eisenegger C, Naef M, Linssen A, Clark L, Gandamaneni PK, Müller U, et al. (2014). Role of dopamine D2 receptors in human reinforcement learning. Neuropsychopharmacology 39: 2366-2375.

Everitt BJ, \& Robbins TW (2005). Neural systems of reinforcement for drug addiction: from actions to habits to compulsion. Nat Neurosci 8: 1481-1489.

Evers EA, Cools R, Clark L, van der Veen FM, Jolles J, Sahakian BJ, et al. (2005). Serotonergic modulation of prefrontal cortex during negative feedback in probabilistic reversal learning. Neuropsychopharmacology 30: 1138-1147.

Feil J, \& Hasking P (2008). The relationship between personality, coping strategies and alcohol use. Addiction Research \& Theory 16: 526-537.

Field M, \& Cox WM (2008). Attentional bias in addictive behaviors: a review of its development, causes, and consequences. Drug and alcohol dependence 97: 1-20.

Fischer AG, \& Ullsperger M (2017). An update on the role of serotonin and its interplay with dopamine for reward. Frontiers in human neuroscience 11: 484.

Floyd FJ, \& Widaman KF (1995). Factor analysis in the development and refinement of clinical assessment instruments. Psychological assessment 7: 286.

Fowles DC (1988). Psychophysiology and psychopathology: A motivational approach. Psychophysiology 25: 373-391.

Frank MJ (2005). Dynamic dopamine modulation in the basal ganglia: a neurocomputational account of cognitive deficits in medicated and nonmedicated Parkinsonism. J Cogn Neurosci 17: 51-72.

Frank MJ, Seeberger LC, \& O'Reilly R C (2004). By carrot or by stick: cognitive reinforcement learning in parkinsonism. Science 306:1940-1943. 
Franken IHA (2002). Behavioral approach system (BAS) sensitivity predicts alcohol craving. Personality and Individual Differences 32: 349-355.

Franken IHA, \& Muris P (2006). BIS/BAS personality characteristics and college students' substance use. Personality and Individual Differences 40: 1497-1503.

Fromme K, \& D'Amico EJ (2000). Measuring adolescent alcohol outcome expectancies. Psychology of addictive behaviors 14: 206.

Fromme K, Stroot EA, \& Kaplan D (1993). Comprehensive effects of alcohol: Development and psychometric assessment of a new expectancy questionnaire. Psychological Assessment 5: 19-26.

Goldman MS (1994). The alcohol expectancy concept: Applications to assessment, prevention, and treatment of alcohol abuse. Applied and Preventive Psychology 3: 131-144.

Golebiowska J, \& Rygula R (2017). Lesions of the Orbitofrontal but Not Medial Prefrontal Cortex Affect Cognitive Judgment Bias in Rats. Front Behav Neurosci 11: 51.

Gorfein DS, \& Weingartner KM (2008). On the norming of homophones. Behavior Research Methods 40: $522-530$.

Grace AA, \& Rosenkranz JA (2002). Regulation of conditioned responses of basolateral amygdala neurons. Physiology \& behavior 77:489-493.

Groman SM, Smith NJ, Petrulli JR, Massi B, Chen L, Ropchan J, et al. (2016). Dopamine D3 Receptor Availability Is Associated with Inflexible Decision Making. J Neurosci 36: 6732-6741.

Grospe GM, Baker PM, \& Ragozzino ME (2018). Cognitive Flexibility Deficits Following 6-OHDA Lesions of the Rat Dorsomedial Striatum. Neuroscience 374: 80-90.

Hales CA, Houghton CJ, \& Robinson ES (2017). Behavioural and computational methods reveal differential effects for how delayed and rapid onset antidepressants effect decision making in rats. European neuropsychopharmacology 27: 1268-1280.

Hasking PA (2006). Reinforcement sensitivity, coping, disordered eating and drinking behaviour in adolescents. Personality and Individual Differences 40: 677-688.

Hindash AHC, \& Amir N (2012). Negative interpretation bias in individuals with depressive symptoms. Cognitive therapy and research 36: 502-511.

Hundt NE, Kimbrel NA, Mitchell JT, \& Nelson-Gray RO (2008). High BAS, but not low BIS, predicts externalizing symptoms in adults. Personality and Individual Differences 44: 565-575.

Iaboni F, Douglas VI, \& Ditto B (1997). Psychophysiological response of ADHD children to reward and extinction. Psychophysiology 34:116-123.

Ineichen C, Sigrist H, Spinelli S, Lesch KP, Sautter E, Seifritz E, et al. (2012). Establishing a probabilistic reversal learning test in mice: evidence for the processes mediating reward-stay and punishment-shift behaviour and for their modulation by serotonin. Neuropharmacology 63: 1012-1021.

Jessup MA, Ross TB, Jones AL, Satre DD, Weisner CM, Chi FW, et al. (2014). Significant life events and their impact on alcohol and drug use: a qualitative study. Journal of psychoactive drugs 46: 450-459.

Jocham G, Klein TA, Neumann J, von Cramon DY, Reuter M, \& Ullsperger M (2009). Dopamine DRD2 polymorphism alters reversal learning and associated neural activity. J Neurosci 29: 3695-3704.

Jones BT, Corbin W, \& Fromme K (2001). Half full or half empty, the glass still does not satisfactorily quench the thirst for knowledge on alcohol expectancies as a mechanism of change.

Kambouropoulos N, \& Staiger PK (2001). The influence of sensitivity to reward on reactivity to alcoholrelated cues. Addiction 96:1175-1185. 
Kellough JL, Beevers CG, Ellis AJ, \& Wells TT (2008). Time course of selective attention in clinically depressed young adults: an eye tracking study. Behav Res Ther 46: 1238-1243.

Kimbrel NA, Nelson-Gray RO, \& Mitchell JT (2007). Reinforcement sensitivity and maternal style as predictors of psychopathology. Personality and Individual Differences 42: 1139-1149.

Klein TA, Neumann J, Reuter M, Hennig J, von Cramon DY, \& Ullsperger M (2007). Genetically determined differences in learning from errors. Science 318: 1642-1645.

Knyazev GG, Slobodskaya HR, Kharchenko II, \& Wilson GD (2004). Personality and substance use in Russian youths: The predictive and moderating role of behavioural activation and gender. Personality and Individual Differences 37: 827-843.

Koob GF (2003). Alcoholism: allostasis and beyond. Alcohol Clin Exp Res 27: 232-243.

Koob GF, \& Volkow ND (2010). Neurocircuitry of addiction. Neuropsychopharmacology 35: 217-238.

Lee CM, Maggs JL, Neighbors C, \& Patrick ME (2011). Positive and negative alcohol-related consequences: associations with past drinking. J Adolesc 34: 87-94.

Leeman RF, Toll BA, Taylor LA, \& Volpicelli JR (2009). Alcohol-induced disinhibition expectancies and impaired control as prospective predictors of problem drinking in undergraduates. Psychology of Addictive Behaviors 23: 553 .

Leigh BC (1989). In search of the seven dwarves: Issues of measurement and meaning in alcohol expectancy research. Psychological bulletin 105: 361.

Loxton NJ, \& Dawe S (2001). Alcohol abuse and dysfunctional eating in adolescent girls: The influence of individual differences in sensitivity to reward and punishment. International Journal of Eating Disorders 29: 455-462.

Mann K, Roos CR, Hoffmann S, Nakovics H, Leménager T, Heinz A, et al. (2018). Precision medicine in alcohol dependence: a controlled trial testing pharmacotherapy response among reward and relief drinking phenotypes. Neuropsychopharmacology 43: 891-899.

Marlatt GA, \& Donovan DM (2005) Relapse prevention: Maintenance strategies in the treatment of addictive behaviors . Guilford press.

McNaughton N, \& Gray JA (2000). Anxiolytic action on the behavioural inhibition system implies multiple types of arousal contribute to anxiety. Journal of affective disorders 61: 161-176.

Moustafa AA, Cohen MX, Sherman SJ, \& Frank MJ (2008). A role for dopamine in temporal decision making and reward maximization in parkinsonism. J Neurosci 28: 12294-12304.

Nishitani N, Nagayasu K, Asaoka N, Yamashiro M, Shirakawa H, Nakagawa T, et al. (2014). Raphe AMPA receptors and nicotinic acetylcholine receptors mediate ketamine-induced serotonin release in the rat prefrontal cortex. International Journal of Neuropsychopharmacology 17: 1321-1326.

Norman P, Boer H, \& Seydel ER (2005). Protection motivation theory. Predicting health behaviour 81: 126.

Noworyta K, \& Rygula R (2021). Phenotypes of reinforcement sensitivity as predictors of the response to acute antidepressant treatment in rats. European Neuropsychopharmacology 43: 102-115.

Noworyta-Sokolowska K, Kozub A, Jablonska J, Rodriguez Parkitna J, Drozd R, \& Rygula R (2019). Sensitivity to negative and positive feedback as a stable and enduring behavioural trait in rats. Psychopharmacology (Berl) 236: 2389-2403.

O'Connor MJ, \& Paley B (2009). Psychiatric conditions associated with prenatal alcohol exposure. Dev Disabil Res Rev 15: 225-234. 
O'Connor RM, \& Colder CR (2005). Predicting Alcohol Patterns in First-Year College Students Through Motivational Systems and Reasons for Drinking. Psychology of Addictive Behaviors 19: 10-20.

O'Connor RM, Stewart SH, \& Watt MC (2009). Distinguishing BAS risk for university students' drinking, smoking, and gambling behaviors. Personality and Individual Differences 46: 514-519.

Ooteman W, Koeter M, Verheul R, Schippers G, \& Van den Brink W (2006). Development and validation of the Amsterdam Motives for Drinking Scale (AMDS): an attempt to distinguish relief and reward drinkers. Alcohol Alcohol 41: 284-292.

Pabst A, Kraus L, Piontek D, \& Mueller S (2010). Age, period, and cohort effects on time trends in alcohol consumption in the German adult population. Sucht.

Papciak J, \& Rygula R (2017). Measuring Cognitive Judgement Bias in Rats Using the Ambiguous-Cue Interpretation Test. Current protocols in neuroscience / editorial board, Jacqueline N Crawley [et al] 78: 9 $5751-595722$.

Pardo Y, Aguilar R, Molinuevo B, \& Torrubia R (2007). Alcohol use as a behavioural sign of disinhibition: Evidence from JA Gray's model of personality. Addictive behaviors 32: 2398-2403.

Paulus MP, Hozack N, Frank L, \& Brown GG (2002). Error rate and outcome predictability affect neural activation in prefrontal cortex and anterior cingulate during decision-making. Neuroimage 15:836-846.

Paulus MP, Hozack N, Frank L, Brown GG, \& Schuckit MA (2003). Decision making by methamphetaminedependent subjects is associated with error-rate-independent decrease in prefrontal and parietal activation. Biol Psychiatry 53: 65-74.

Pessiglione M, Seymour B, Flandin G, Dolan RJ, \& Frith CD (2006). Dopamine-dependent prediction errors underpin reward-seeking behaviour in humans. Nature 442: 1042-1045.

Peterson C, Semmel A, Von Baeyer C, Abramson LY, Metalsky GI, \& Seligman ME (1982). The attributional style questionnaire. Cognitive therapy and research 6: 287-299.

Robinson TE, \& Berridge KC (1993). The Neural Basis of Drug Craving - an Incentive-Sensitization Theory of Addiction. Brain Res Rev 18: 247-291.

Roos CR, Mann K, \& Witkiewitz K (2017). Reward and relief dimensions of temptation to drink: construct validity and role in predicting differential benefit from acamprosate and naltrexone. Addiction biology 22: 1528-1539.

Rychlik M, Bollen E, \& Rygula R (2017). Ketamine decreases sensitivity of male rats to misleading negative feedback in a probabilistic reversal-learning task. Psychopharmacology (Berl) 234: 613-620.

Rygula R, Clarke HF, Cardinal RN, Cockcroft GJ, Xia J, Dalley JW, et al. (2015). Role of Central Serotonin in Anticipation of Rewarding and Punishing Outcomes: Effects of Selective Amygdala or Orbitofrontal 5-HT Depletion. Cereb Cortex 25: 3064-3076.

Rygula R, Noworyta-Sokolowska K, Drozd R, \& Kozub A (2018). Using rodents to model abnormal sensitivity to feedback in depression. Neurosci Biobehav Rev 95: 336-346.

Rygula R, Papciak J, \& Popik P (2014). The effects of acute pharmacological stimulation of the 5-HT, $\mathrm{NA}$ and DA systems on the cognitive judgement bias of rats in the ambiguous-cue interpretation paradigm. European neuropsychopharmacology : the journal of the European College of Neuropsychopharmacology 24: 1103-1111.

Rygula R, \& Popik P (2016). Trait "pessimism" is associated with increased sensitivity to negative feedback in rats. Cogn Affect Behav Neurosci 16: 516-526. 
Sachs BD, Rodriguiz RM, Tran HL, Iyer A, Wetsel WC, \& Caron MG (2015). Serotonin deficiency alters susceptibility to the long-term consequences of adverse early life experience. Psychoneuroendocrinology 53:6981.

Samson RD, Frank MJ, \& Fellous J-M (2010). Computational models of reinforcement learning: the role of dopamine as a reward signal. Cognitive Neurodynamics 4: 91-105.

Schaefer KL, Baumann J, Rich BA, Luckenbaugh DA, \& Zarate Jr CA (2010). Perception of facial emotion in adults with bipolar or unipolar depression and controls. Journal of Psychiatric Research 44:1229-1235.

Scheier MF, \& Carver CS (2018). Dispositional optimism and physical health: A long look back, a quick look forward. American Psychologist 73: 1082.

Schick A, Wessa M, Vollmayr B, Kuehner C, \& Kanske P (2013). Indirect assessment of an interpretation bias in humans: neurophysiological and behavioral correlates. Frontiers in human neuroscience 7: 272.

Schulman P, Castellon C, \& Seligman ME (1989). Assessing explanatory style: The content analysis of verbatim explanations and the Attributional Style Questionnaire. Behaviour research and therapy 27: 505509.

Sharot T, Guitart-Masip M, Korn CW, Chowdhury R, \& Dolan RJ (2012). How dopamine enhances an optimism bias in humans. Curr Biol 22:1477-1481.

Sharot T, Korn CW, \& Dolan RJ (2011). How unrealistic optimism is maintained in the face of reality. Nature neuroscience 14:1475-1479.

Sharot T, Shiner T, Brown AC, Fan J, \& Dolan RJ (2009). Dopamine enhances expectation of pleasure in humans. Curr Biol 19:2077-2080.

Shepperd JA, Klein WM, Waters EA, \& Weinstein ND (2013). Taking Stock of Unrealistic Optimism. Perspect Psychol Sci 8: 395-411.

Skinner MD, \& Aubin HJ (2010). Craving's place in addiction theory: contributions of the major models. Neurosci Biobehav Rev 34:606-623.

Slaney C, Hinchcliffe JK, \& Robinson ESJ (2018). Translational Shifts in Preclinical Models of Depression: Implications for Biomarkers for Improved Treatments. Curr Top Behav Neurosci 40: 169-193.

Swainson R, Rogers RD, Sahakian BJ, Summers BA, Polkey CE, \& Robbins TW (2000). Probabilistic learning and reversal deficits in patients with Parkinson's disease or frontal or temporal lobe lesions: possible adverse effects of dopaminergic medication. Neuropsychologia 38: 596-612.

Tranel DT (1983). The Effects of Monetary Incentive and Frustrative Nonreward on Heart Rate and Electrodermal Activity. Psychophysiology 20: 652-657.

Voigt K, Twork S, Mittag D, Göbel A, Voigt R, Klewer J, et al.(2009). Consumption of alcohol, cigarettes and illegal substances among physicians and medical students in Brandenburg and Saxony (Germany). BMC Health Serv Res 9: 219.

Walton MA, Castro FG, \& Barrington EH (1994). The role of attributions in abstinence, lapse, and relapse following substance abuse treatment. Addict Behav 19: 319-331.

Wardell JD, O'Connor RM, Read JP, \& Colder CR (2011). Behavioral approach system moderates the prospective association between the behavioral inhibition system and alcohol outcomes in college students. J Stud Alcohol Drugs 72: 1028-1036.

Warren RE, Warren NT, Green JP, \& Bresnick JH (1978). Multiple semantic encoding of homophones and homographs in contexts biasing dominant or subordinate meanings. Memory \& Cognition 6: 364-371. 
Weiner B (1985). An attributional theory of achievement motivation and emotion. Psychological Review 92: 548-573.

Wilkinson MP, Grogan JP, Mellor JR, \& Robinson ES (2020). Comparison of conventional and rapidacting antidepressants in a rodent probabilistic reversal learning task. Brain and neuroscience advances $4: 2398212820907177$.

Willeit M, \& Praschak-Rieder N (2010). Imaging the effects of genetic polymorphisms on radioligand binding in the living human brain: A review on genetic neuroreceptor imaging of monoaminergic systems in psychiatry. Neuroimage 53: 878-892.

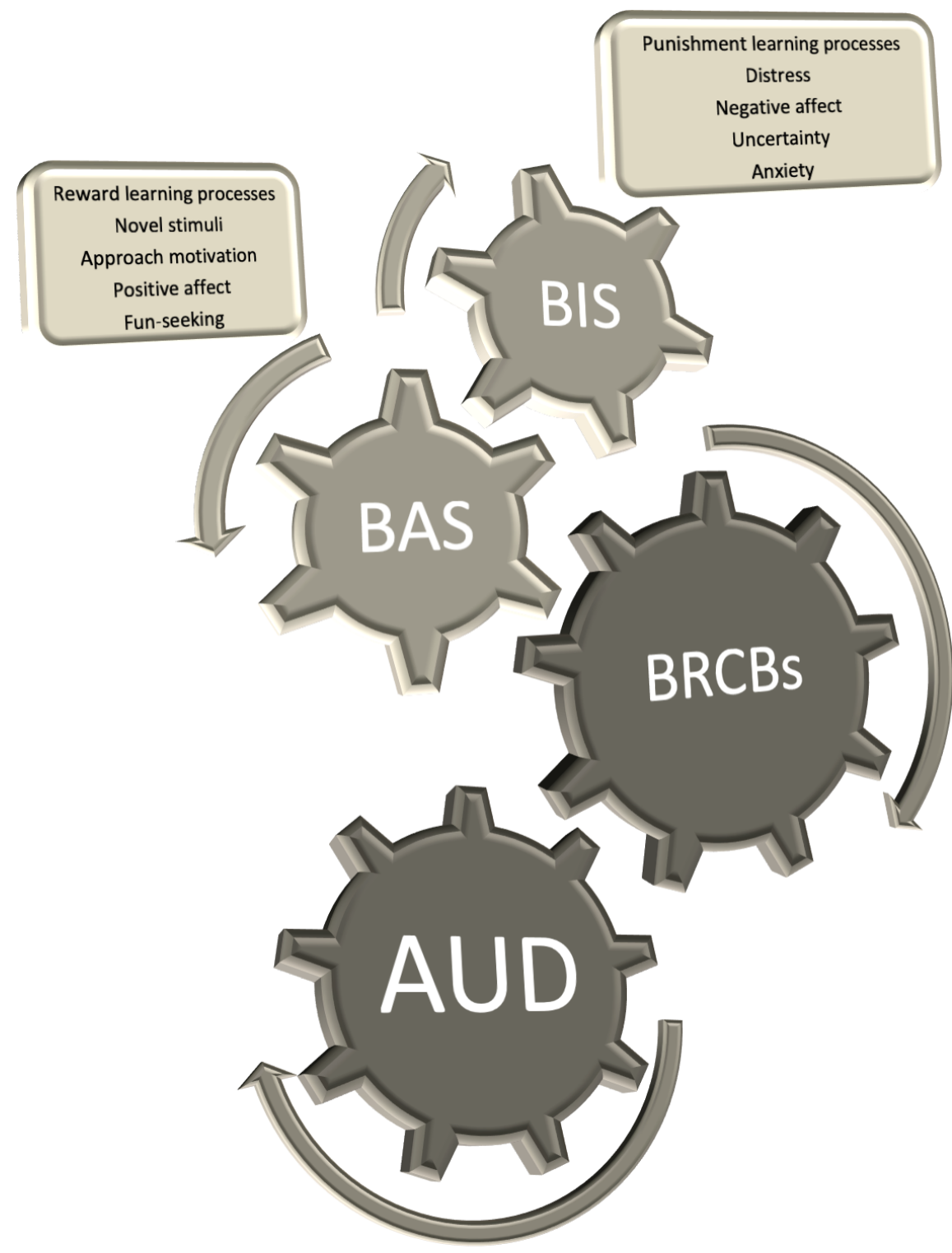




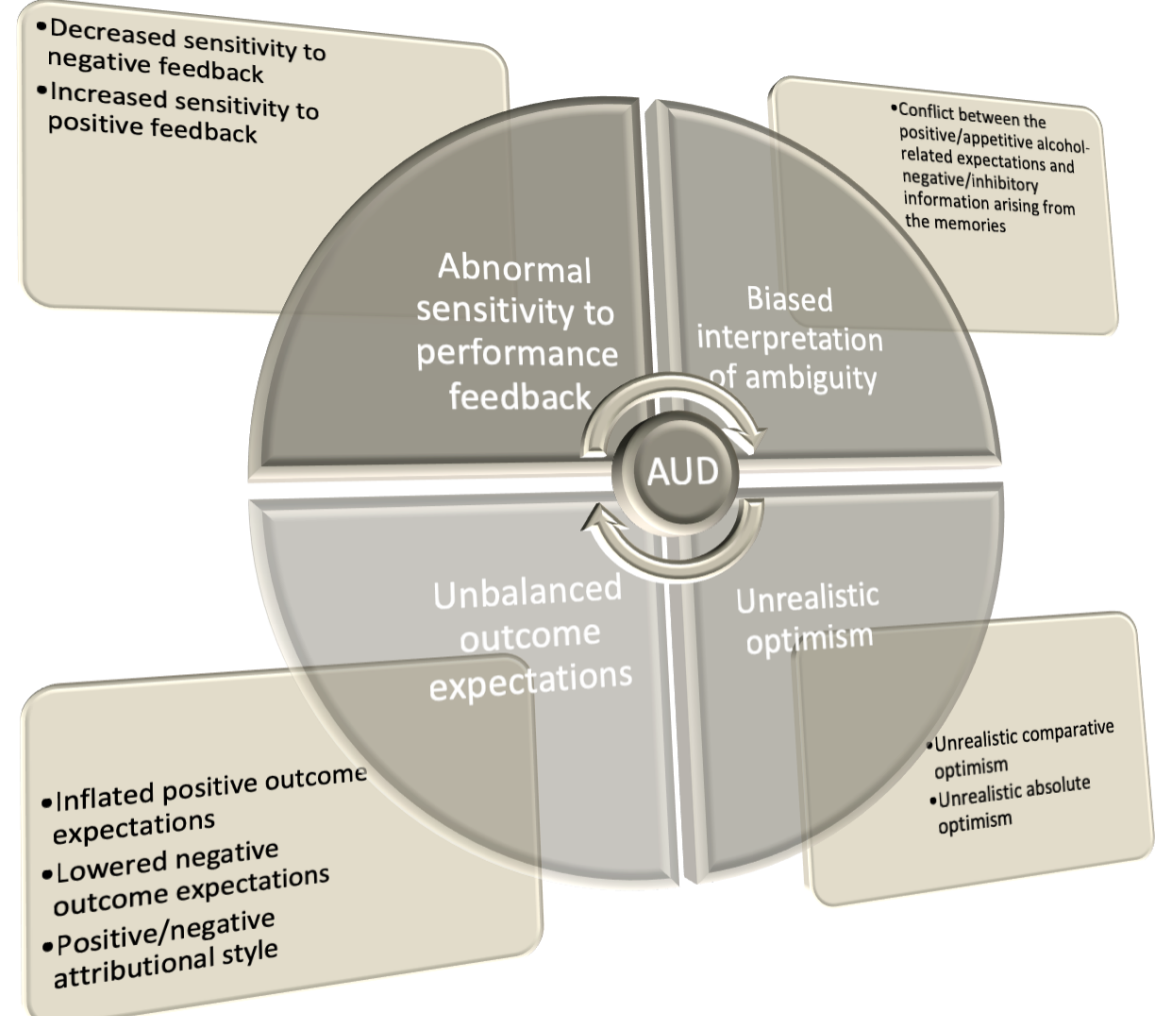




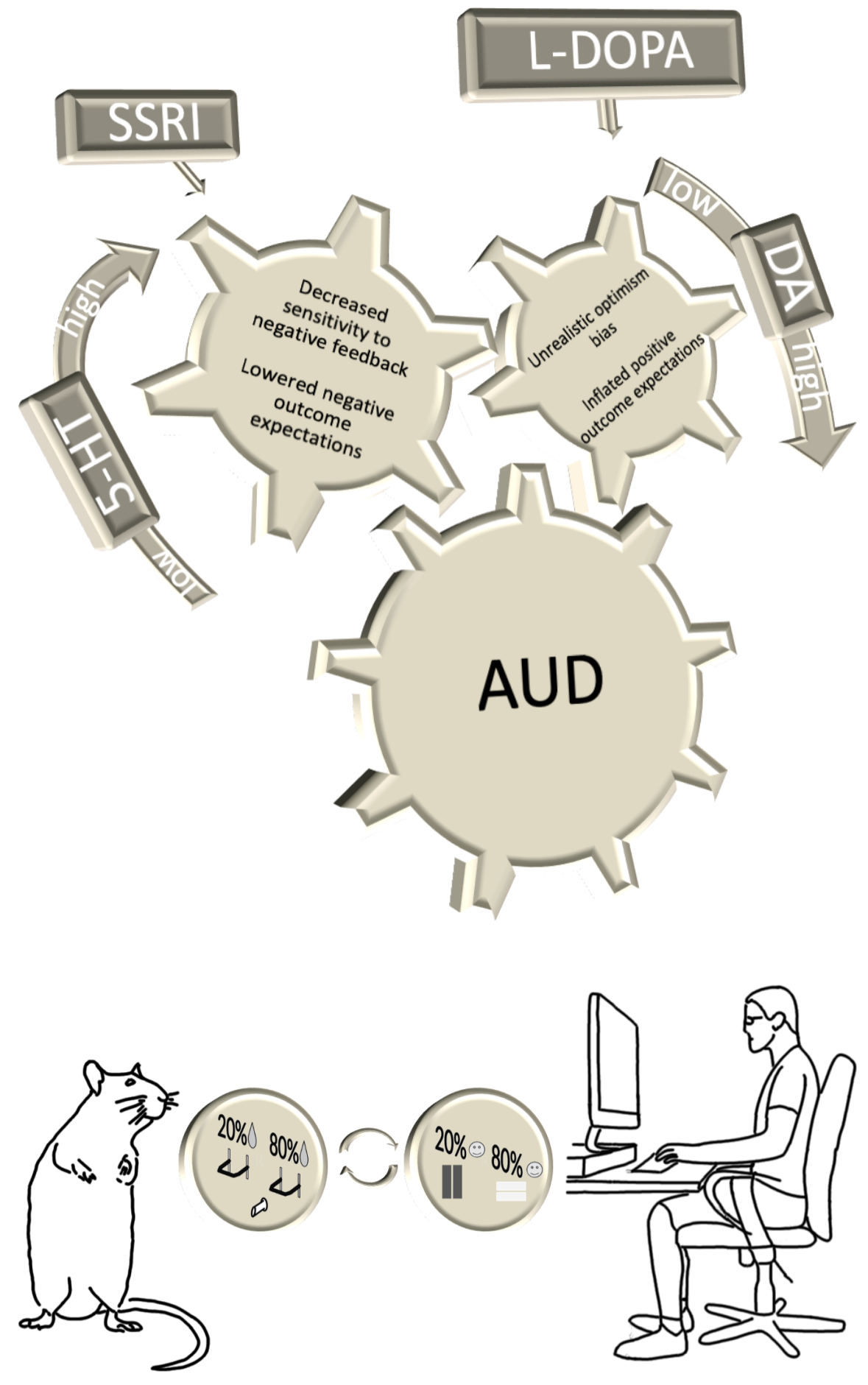

